\title{
A Simple, Inexpensive Method for Using Unmanned Aerial Vehicle Photograph Analysis to Quantify Green Color and Enhance Ratings in Field Research Plots
}

\author{
Trey Price, ${ }^{1,+}$ Sebe Brown, ${ }^{2}$ and Randy Price ${ }^{2}$ \\ ${ }^{1}$ LSU AgCenter, Macon Ridge Research Station, Winnsboro, LA 71295 \\ ${ }^{2}$ LSU AgCenter, Dean Lee Research and Extension Center, Alexandria, LA 71302
}

Accepted for publication 21 May 2019.

Keywords: foliar diseases, foliar insect pests, integrated pest management, precision agriculture, field crops

Agricultural small-plot research is crucial for developing integrated pest management solutions for stakeholders and is carried out at land grant university research stations, private contract research farms, and producers' fields. Small-plot research experiments include foliar or seed treatment pesticide efficacy trials or multientry plant variety trials in which investigators determine the effectiveness of experimental and commercially available products against specific pests in crops.

Evaluations involve rating for disease or damage using scales requiring visual estimation. Humans can introduce considerable variability into these estimations. To combat the variability introduced by human perception, many researchers have attempted to incorporate computer-generated scales that more accurately measure affected areas of foliage (Del Ponte et al. 2017). Other efforts to remotely detect biotic and abiotic stresses have included techniques such as satellite imagery, fluorescence, normalized difference vegetation index (NDVI), and thermography (Mirik et al. 2011; Sandmann et al. 2018). Developing meaningful remotely sensed values from aerial imagery may involve a considerable amount of time and resources, luxuries that many public and private researchers may not have. Recent developments in unmanned aerial vehicle (UAV) technology have allowed novices to acquire aerial imagery with cameras that can be modified to filter infrared and red color wavelengths and allow for the quantification of reflected light within specific color spectra. The objective of this study was to derive quantifiable data from aerial photographs of research plots and compare with data acquired by humans at ground level.

Small-plot soybean fungicide efficacy trials were established at the Macon Ridge and Dean Lee Research Stations near Winnsboro and Alexandria, LA, during the 2017 (four trials, 130 total plots) and 2018 (four trials, 108 total plots) growing seasons, respectively. All trials were conducted in randomized complete block designs with four replications. Commercial and/or experimental fungicide treatments were applied at R3 and/or R5. Plots in all trials had varying degrees of Cercospora leaf blight (CLB) (Cercospora spp.) severity and were rated visually using a 1 to 9 scale (Padgett and Purvis 2008). On the same day, a relatively inexpensive $(<\$ 1,000)$ DJI (Shenzhen, China) Phantom 3 Professional drone outfitted with a camera unit advertised

${ }^{\dagger}$ Corresponding author: T. Price; E-mail: pprice@agcenter.lsu.edu

The author(s) declare no conflict of interest.

(C) 2019 The American Phytopathological Society as NDVI (Aerial Media Pros, Costa Mesa, CA) was used to acquire a snapshot aerial photograph of entire test areas. The camera provided a near infrared/red filtered image that appears pink with lighter-colored areas indicating more green foliage (Fig. 1). Photographs were analyzed using the free Gnu Image Manipulation Program (GIMP, https:// www.gimp.org/) version 2.8.10. Using the rectangular selection tool, the interior portion of a plot was selected to avoid plot edges (Fig. 2). The histogram option was used to generate a mean value of the green channel, which averaged all of the green wavelength values for the number of selected pixels. The selected rectangular area remained the same size when moved from plot to plot in a given photograph, and individual mean green values were recorded (Fig. 2). Wavelength values from the camera existed in an 8-bit resolution that ranged from 0 to 255 in value. The aperture was left in an automatic float mode to center light levels during the flight. The CLB rating data were compared with remotely sensed data using Pearson correlation $(\alpha=$ 0.05 ). Data were transformed using arcsine square root of $X+0.5$ to satisfy variance homogeneity, if necessary.

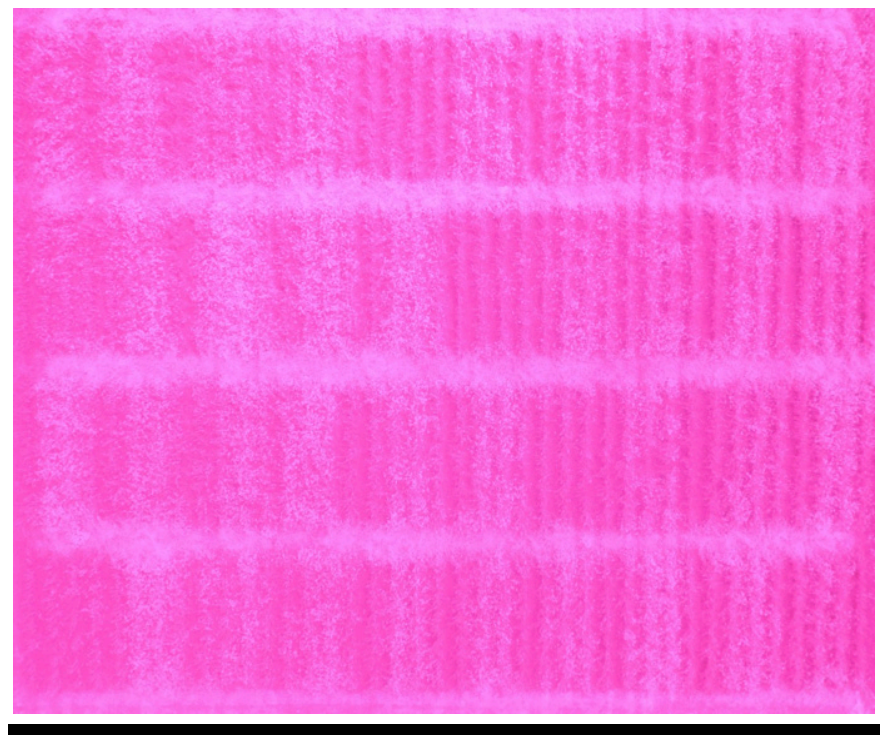

FIGURE 1

A near infrared/red filtered image of a test area with varying degrees of defoliation caused by Cercospora leaf blight. Lighter pink color indicates more green foliage within fungicide-treated plots. 


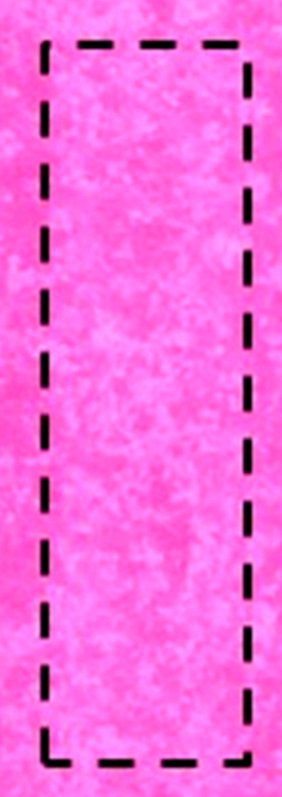

\section{Fungicide-treated}

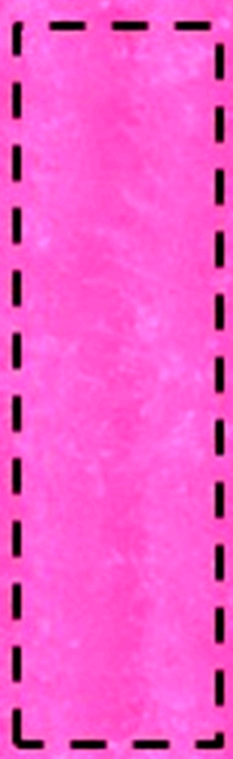

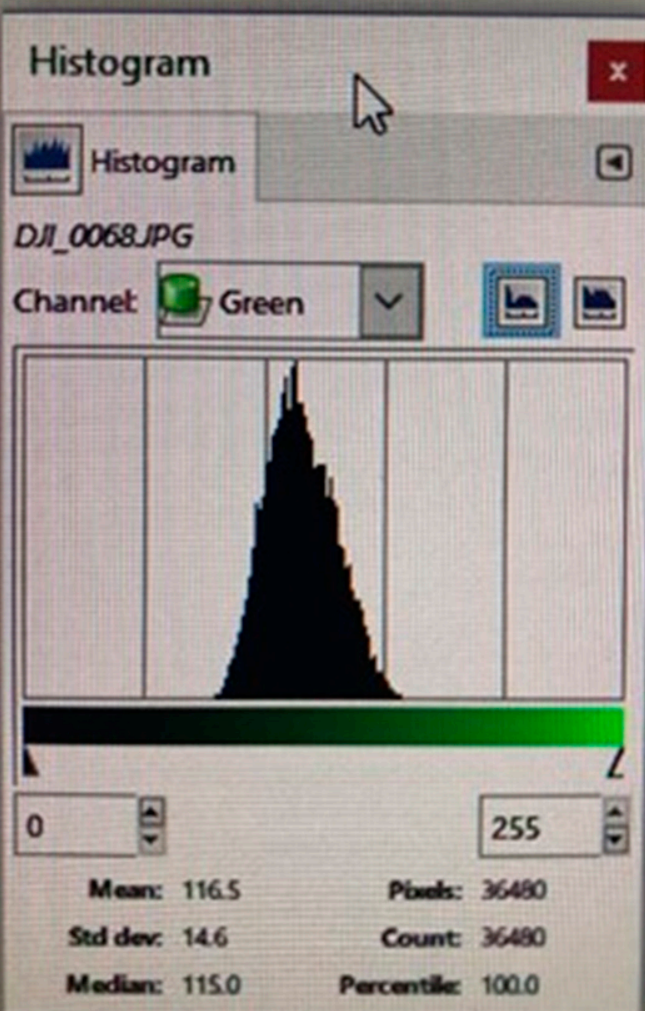

Non-treated

\section{Histogram}

Histogram

๑

DI_0068JPG
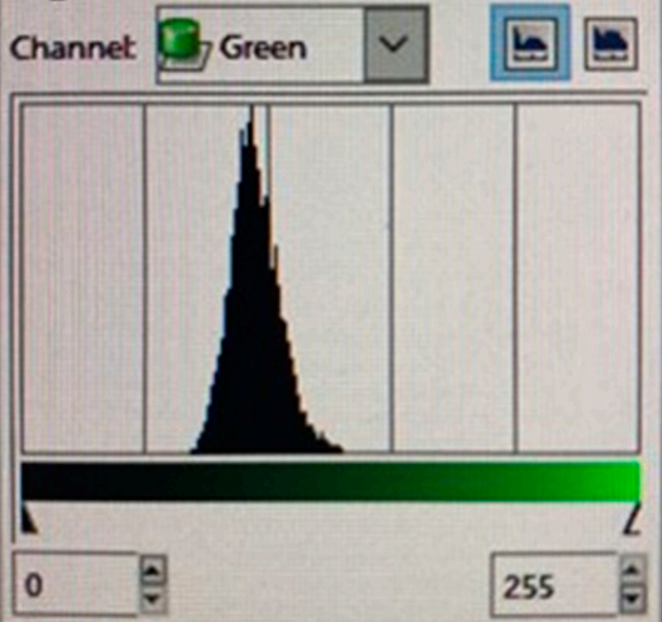

Mean: 961

Pbeds: 36400

Std dev: 10.4

Count 36490

Median: 950

FIGURE 2

Quantifying green color in fungicide-treated (upper) and nontreated (lower) soybean plots affected by Cercospora leaf blight (Cercospora spp.) using Gnu Image Manipulation Program (GIMP) version 2.8.10. Histograms along with mean, standard deviation, median, pixels, count, and percentile are generated by GIMP for the outlined area. 
TABLE 1

\begin{tabular}{|c|c|c|c|c|}
\hline Trial location-year (no.) & $P$ value & $R^{2}(r)$ & CLB rating CV & Green color value CV \\
\hline Winnsboro-2017 (2) & 0.0003 & $0.3595(-0.5996)$ & 9.23 & 1.23 \\
\hline Winnsboro-2017 (3) & 0.0001 & $0.5348(-0.7313)$ & 9.59 & 1.39 \\
\hline Winnsboro-2017 (4) & 0.0001 & $0.6315(-0.7947)$ & 9.49 & 2.19 \\
\hline Alexandria-2018 (3) & 0.0040 & $0.1812(-0.4256)$ & 20.36 & 11.79 \\
\hline Alexandria-2018 (4) & 0.0190 & $0.2694(-0.5190)$ & 17.84 & 9.24 \\
\hline
\end{tabular}

${ }^{\mathrm{a}} \mathrm{CV}=$ coefficient of variation.

Green color and rating values were significantly and negatively correlated in all eight trials (Table 1). That is, plots with the most disease had the lowest green values and vice versa. Furthermore, the relative variability of the remotely sensed data was considerably lower when compared with CLB rating data (Table 1).

This method may allow relatively quick, inexpensive, and simple generation of remotely sensed data that can improve, enhance, and/ or confirm data in small-plot research soybean trials. Because the correlated wavelength was green, standard UAVs with unmodified cameras may be useful for this operation. Applications of this method may exist for other foliar diseases, foliar insect pests, and ground cover estimations in multiple crops.

\section{Literature Cited}

Del Ponte, E. M., Pethybridge, S. J., Bock, C. H., Michereff, S. J., Machado, F. J., and Spolti, P. 2017. Standard area diagrams for aiding severity estimation: Scientometrics, pathosystems, and methodological trends in the last 25 years. Phytopathology 107:1161-1174.

Mirik, M., Jones, D. C., Price, J. A., Workneh, F., Ansley, R. J., and Rush, C. M. 2011. Satellite remote sensing of wheat infected by wheat streak mosaic virus. Plant Dis. 95:4-12.

Padgett, G. B., and Purvis, M. A. 2008. Selected fungicide treatments for managing soybean rust and Cercospora foliar blight, 2007. Plant Dis. Manage. Rep. 2:FC046.

Sandmann, M., Grosch, R., and Graefe, J. 2018. The use of features from fluorescence, thermography, and NDVI imaging to detect biotic stress in lettuce. Plant Dis. 102:1101-1107. 